\title{
Adipose stem cells fail to boost cartilage repair in rats
}

Adipose stem cells (ASCs), although multipotent, might not offer the route to cartilage repair that had been hoped, at least not without development of a pretreatment regimen. Barbara Boyan and colleagues found that ASCs secrete angiogenic factors that induce chondrocyte apoptosis in vitro, and inhibit cartilage regeneration in vivo.

\section{4 ...[ASCs] produce factors associated with wound repair and angiogenesis 77}

Traumatic injuries to cartilage heal with noncartilaginous tissue, and as chondrocytes cannot be isolated from human donors in large numbers, the researchers attempted to use more readily available ASCs to stimulate cartilage regeneration. In culture, these cells produce factors associated with wound repair and angiogenesis. "Cartilage is an avascular tissue, suggesting that an angiogenic signal would not be appropriate when chondrogenesis was the intent" explains Boyan. "However, it was possible that ASCs in a chondral site might receive information from the surrounding tissue that would mitigate the angiogenic response."

Initial in vitro experiments showed that rat ASCs produced large amounts of vascular endothelial growth factor A (VEGF-A). Factors secreted by ASCs inhibited proliferation of chondrocytes and increased their apoptosis. However, treating ASCs with chondrogenic medium substantially reduced VEGF-A secretion and the deleterious effects of the secreted factors on chondrocytes.

The effect of ASCs on cartilage regeneration was assessed in vivo using a rat model. The cells were implanted into defects in chondral cartilage located at the base of the sternum, an area chosen because the tissue remains as cartilage throughout the life of the animal. 35 days after surgery, ASCs that were cultured in growth medium prior to implantation inhibited cartilage regeneration, whereas those that were cultured in chondrogenic medium had no effect on repair; the degree of healing was not significantly different from that of defects that were not implanted with ASCs.

Boyan thinks that optimization of pretreatment regimens and delivery methods might make it possible to use ASCs to treat chondral defects. However, the researchers are now investigating "how to best take advantage of the ability of ASCs to generate angiogenic factors to treat clinical conditions where angiogenesis is desired".

Ellen F. Carney

Original article Lee, C. S. et al. Adipose stem cells can secrete angiogenic factors that inhibit hyaline cartilage regeneration. Stem Cell Res. Ther. doi:10.1186/scrt126 